\title{
A CLASS OF MIX DESIGN PROBLEMS: FORMULATION, SOLUTION METHODS AND APPLICATIONS
}

\author{
ZHONG WAN ${ }^{1}$, K. L. TEO ${ }^{\bowtie 2},{\text { LINGSHUANG } \text { KONG }^{3} \text { and CHUNHUA YANG }}^{3}$
}

(Received 16 January, 2009; revised 2 April, 2009)

\begin{abstract}
In this paper, we consider a linear program with only equality constraints but containing interval and random coefficients. We first address the linear program with interval coefficients, and establish some structural properties of this linear program. On this basis, a solution method is proposed. We then move on to consider the linear program with random coefficients. Using the chance constraint approach and a new approach, the satisfaction degree approach, we obtain the two respective deterministic equivalent formulations. Then the results and the numerical solution methods obtained for these two linear models are applied to the original linear problem which contains both interval and random coefficients. By way of illustration, we consider a practical problem, where the optimal mixing proportions need to be determined for the mix slurry in the production process of aluminium with sintering. This gives rise to a linear program with interval and random coefficients. Its deterministic equivalent formulations are presented. Preliminary numerical examples show that the proposed models and the solution methods are promising.
\end{abstract}

2000 Mathematics subject classification: primary 62K05; secondary 90C70, 49N10.

Keywords and phrases: linear programming, random linear programs, chance constrained programming, linear programming with interval coefficients, mixture making, alumina sintering.

\section{Introduction}

In the past two decades, mix design problems have attracted much attention from process engineers, researchers and key executives from government and various industries due to the importance of efficient resource utilization. Some mathematical

\footnotetext{
${ }^{1}$ School of Mathematical Science and Computing Technology, Central South University, Hunan Changsha, PR China; e-mail: wanmath@163.com.

${ }^{2}$ Department of Mathematics and Statistics, Curtin University of Technology, Perth, Australia; e-mail: K.L.Teo@curtin.edu.au.

${ }^{3}$ School of Information Sciences and Engineering, Central South University, Hunan Changsha, PR China.

(C) Australian Mathematical Society 2009, Serial-fee code 1446-1811/2009 \$16.00
} 
models and solution methods have been constructed and developed (see [1, 4, 10$12,15,20,22]$ and the references therein). These models mainly focus on mixing processes without random parameters. However, the occurrence of uncertainty is unavoidable in practice. Thus, such deterministic formulations are inadequate. In this paper, we will develop a new general formulation for a class of mixing problems, and develop some efficient solution methods.

For each $k \in\{1,2, \ldots, r\}$ and $j \in\{1,2, \ldots, s\}$, let $p_{k j}$ denote the amount of the $j$ th ingredient in the $k$ th raw material. Clearly, $p_{k j} \geq 0$. The matrix $P=\left(p_{k j}\right) \in R^{r \times s}$ is called an ingredient matrix. Assuming that no chemical reaction will take place during the mixing process, the mixture design problem is to find optimal mixing proportions of the given raw materials such that some desired product qualities are achieved. A simple case of such a mixture problem is the mixture design of high-performance concrete in building engineering. For studies of such problems, see $[4,10,12,15,20,25]$ and the references cited therein. However, the mixture making problems encountered in metallurgical engineering are much more complex. For example, due to uncertain qualities of some raw materials, it is often difficult to obtain a mixing scheme that guarantees a consistent product quality.

To find the optimal scheme for the aforementioned mixture problem, a common approach is to formulate it as an optimization problem (see [1, 12, 21, 22]), where the objective is to minimize a cost function for the raw materials to be processed, such as mulling the ore in the metallurgical industry, subject to the constraints of maintaining product quality specifications. It appears that linear programming is the most commonly used approach for solving these problems. For other algorithms, see $[1,12,22]$. The main deficiency of these existing methods is that they do not take into consideration of the randomness in the mixing process. It is clear that such omission could have major consequences in a real production. Thus, the nondeterministic parameters should be taken into account in the mathematical formulation of the mixture making.

Motivated by the importance of the practical issues mentioned above and recent developments in the theory and algorithms of random programming and advanced optimization techniques (see [2, 3, 6-9, 13, 16-19]), this paper will formulate the above mixing proportion problems as linear programs with only equality constraints in addition to the box constraints. The equality constraints contain some interval and random coefficients. This formulation is realistic but is much more complicated than the existing ones based on ordinary (random) linear programming [13, 18]. We shall develop a new efficient solution method for this problem.

The remainder of this paper is organized as follows. In Section 2, we will present sufficient and necessary conditions for admissible mixing designs. Then, in Section 3, we will formulate the mixture making problem as a new linear program involving equality constraints in addition to the box constraints, with interval or random coefficients involved in some of these equality constraints. The uncertainty effects during the production process will be taken into consideration, from the construction of models to the development of numerical solution methods. These random effects will be captured through the introduction of interval and random coefficients in the 
constraints. In Section 4, efficient solution methods will be developed by fully utilizing the specific features of the models. In Section 5, we apply the models obtained to a specific real practical problem, where optimal mixing proportions need to be determined for the mix slurry with sintering. A numerical example is reported in Section 6 , followed by some concluding remarks.

\section{Admissibility of mixing design}

Let the total weight $(\mathrm{kg})$ of all raw materials be $M_{0}$, and let $x_{k}, k=1,2, \ldots, r$, be the percentage composition of the $k$ th raw material. Assume that there is no chemical reaction in the mixing process among the components of the raw materials. The total weight of the ingredient $j$ after mixture making is given by the formula

$$
u_{j}=\sum_{k=1}^{r} M_{0} x_{k} p_{k j}, \quad j=1,2, \ldots, s,
$$

where $p_{k j}$ is the amount of the $j$ th ingredient in the $k$ th raw material. Let $u=$ $\left[u_{1}, u_{2}, \ldots, u_{s}\right]^{T}$ and $P=\left(p_{j k}\right)$. Then (2.1) can be written compactly as

$$
u=M_{0} P^{T} x .
$$

Assume that there are a total of $m$ quality indices for the product. Let $f_{i}, i=$ $1,2, \ldots, m$, denote the $i$ th index, which is a function of the variables $u_{1}, u_{2}, \ldots, u_{s}$. In practice, these functions can often be expressed as linear rational functions

$$
f_{i}(u)=\frac{a_{i 1} u_{1}+a_{i 2} u_{2}+\cdots+a_{i s} u_{s}}{b_{i 1} u_{1}+b_{i 2} u_{2}+\cdots+b_{i s} u_{s}}, \quad i=1,2, \ldots, m,
$$

where $a_{i j}$ and $b_{i j}$ are given scalars.

Set $A=\left(a_{i j}\right) \in R^{m \times s}$ and $B=\left(b_{i j}\right) \in R^{m \times s}$. Let $\hat{f}_{i}$ be the optimal value of the $i$ th quality index, where $i=1,2, \ldots, m$. Then the mixing proportions $x_{k}$, $k=1,2, \ldots, r$, which are required to be chosen such that all the quality indices are fulfilled, must satisfy the conditions

$$
f_{i}(u)=\hat{f}_{i}, \quad i=1,2, \ldots, m .
$$

Equation (2.2) can be written in a compact matrix form

$$
(A-\operatorname{diag}(\hat{f}) B) u=0,
$$

where $\hat{f}=\left[\hat{f}_{1}, \hat{f}_{2}, \ldots, \hat{f}_{m}\right]^{T}$. Substituting for $u$ into (2.3) and removing $M_{0}$ in the resulting equation yields

$$
(A-\operatorname{diag}(\hat{f}) B) P^{T} x=0 .
$$

Set

$$
C=(A-\operatorname{diag}(\hat{f}) B) P^{T} .
$$

Then (2.4) can be written as

$$
C x=0 .
$$


Definition 2.1. We call $x=\left[x_{1}, x_{2}, \ldots, x_{r}\right]^{T} \geq 0$ a feasible mixing proportion if it is a solution of the admissibility conditions (2.6).

Clearly, admissibility conditions (2.6) are the standard homogeneous linear algebraic equations.

In the following, we will present some sufficient and necessary conditions for ensuring the existence of nonzero solutions to (2.6). Since these results can be obtained from the basic theory of linear algebra, we omit the proofs.

Proposition 2.2. Let $C=\left(c_{i k}\right)$, where $c_{i k}$ is defined as in (2.5). Then there exists a nonzero solution of (2.6) if and only if $R(C)<\min \{m, r\}$, where $R(C)$ denotes the rank of the matrix $C$.

Corollary 2.3. Let $\alpha_{k} \in \mathbb{R}^{m}, k=1,2, \ldots, r$, denote the $k$ th column of $C$. Then there exists a nonzero solution of (2.6) if and only if the set of the vectors $\left\{\alpha_{1}, \alpha_{2}, \ldots, \alpha_{r}\right\}$ is linearly dependent.

COROLLARY 2.4. If $m<r$, then there exists a nonzero solution of (2.6).

REMARK 2.5. In practice, the condition $m<r$ means that the quality indices cannot be more than the number of distinct raw materials.

Corollary 2.6. Assume that $\min \{m, r\}>1$. If $s=1$, then $R(C) \leq 1$, and hence there exists a nonzero solution of (2.6).

ProOF. Denote

$$
\bar{c}_{i j}=a_{i j}-\hat{f}_{i} b_{i j}
$$

So,

$$
c_{i k}=\sum_{j=1}^{s} \bar{c}_{i j} p_{k j} \triangleq \bar{c}_{i} p_{k}^{T},
$$

where $\bar{c}_{i}=\left[\bar{c}_{i 1}, \ldots, \bar{c}_{i s}\right]$ and $p_{k}=\left[p_{k 1}, \ldots, p_{k s}\right]$.

If $s=1$, then from (2.5), we have

$$
C=\left[\begin{array}{cccc}
\bar{c}_{11} p_{11} & \bar{c}_{11} p_{21} & \cdots & \bar{c}_{11} p_{r 1} \\
\bar{c}_{21} p_{11} & \bar{c}_{21} p_{21} & \cdots & \bar{c}_{21} p_{r 1} \\
\vdots & \vdots & & \vdots \\
\bar{c}_{m 1} p_{11} & \bar{c}_{m 1} p_{21} & \cdots & \bar{c}_{m 1} p_{r 1}
\end{array}\right]
$$

Clearly, $R(C) \leq 1$. Hence, there exists a nonzero solution of (2.6).

Denote $\bar{C}=\left(\bar{c}_{i j}\right) \in \mathbb{R}^{m \times s}$. Then, for $s \geq 1$, it follows from (2.8) that

$$
C=\bar{C} P^{T},
$$

where $P=\left(p_{k j}\right) \in \mathbb{R}^{r \times s}$ is a given ingredient matrix. Therefore, the admissibility conditions (2.6) can be expressed in an alternative form

$$
\bar{C} P^{T} x=0 .
$$

For the linear system (2.10), it is easy to prove the following corollary. 
COROLlaRY 2.7. If $R(\bar{C})<r$, or $R(P)<r$, then there exists a nonzero solution of (2.6).

Finally, from the results presented above and Farkas' lemma, we obtain the following theorem.

THEOREM 2.8 (Admissibility condition).

(1) If $m<r$, then there exists an $x^{*} \in R^{r}$ such that

$$
C x=0, \quad x \geq 0 .
$$

(2) If $R(\bar{C})<r$, or $R(P)<r$, then there exists an $x^{*} \in R^{r}$ such that

$$
\bar{C} P^{T} x=0, \quad x \geq 0 .
$$

\section{Optimization models for the mixing design}

In this section, we will formulate the mixing design problem as a new class of optimization problems.

Let $p_{k}$ denote the price of the $k$ th raw material, where $k=1,2, \ldots, r$. Then the total cost for all the raw materials $M_{0}$ is

$$
f(x)=M_{0} p^{T} x,
$$

where $p=\left[p_{1}, p_{2}, \ldots, p_{r}\right]^{T}$ is a given price vector. By virtue of the discussions in Section 2, the optimal mixing design problem can be described as the linear program

$$
\begin{array}{ll}
\min & f(x) \\
\text { subject to } & \bar{C} P^{T} x=0, \quad \sum_{k=1}^{r} x_{k}=1, \quad l \leq x \leq u,
\end{array}
$$

where $l, u \in \mathbb{R}^{r}$ are, respectively, given lower and upper bounds for $x$. Note that, without loss of generality, $M_{0}$ can be removed from $f$.

Let

$$
A=\left[\begin{array}{c}
C \\
e^{T}
\end{array}\right],
$$

where $C$ is defined in (2.9) and $e$ denotes the vector of ones. Suppose that the rank of the matrix $A$ is $r_{0}$. Then $A x=[0,1]^{T}$ can be rewritten as

$$
\left(A_{B}, A_{N}\right)\left[\begin{array}{l}
x_{B} \\
x_{N}
\end{array}\right]=\left[\begin{array}{l}
0 \\
1
\end{array}\right],
$$

where the matrices $\left(A_{B}, A_{N}\right)$ and $\left(x_{B}, x_{N}\right)$ are the reordering of $A$ and $x$. Here, $A_{B}$ is a nonsingular $r_{0} \times r_{0}$ square submatrix of $A$ and $x_{A}$ consists of the components of $x$ corresponding to the columns of $A_{B}$. From (3.2), we obtain

$$
x_{B}=A_{B}^{-1}\left[\begin{array}{l}
0 \\
1
\end{array}\right]-A_{B}^{-1} A_{N} x_{N} .
$$


Substituting $x_{B}$ into the objective function, Problem (3.1) is reduced to an equivalent lower-dimensional problem

$$
\begin{array}{ll}
\min & \tilde{f}\left(x_{N}\right)=p_{B}^{T}\left[A_{B}^{-1}\left[\begin{array}{l}
0 \\
1
\end{array}\right]-A_{B}^{-1} A_{N} x_{N}\right]+p_{N}^{T} x_{N} \\
\text { subject to } & 0 \leq A_{B}^{-1}\left[\begin{array}{l}
0 \\
1
\end{array}\right]-A_{B}^{-1} A_{N} x_{N} \leq 1, \quad l_{N} \leq x_{N} \leq u_{N} .
\end{array}
$$

We can further simplify (3.3), yielding

$$
\begin{array}{ll}
\min & \tilde{f}\left(x_{N}\right)=\left(p_{N}^{T}-p_{B}^{T} A_{B}^{-1} A_{N}\right) x_{N} \\
\text { subject to } & 0 \leq A_{B}^{-1}\left[\begin{array}{l}
0 \\
1
\end{array}\right]-A_{B}^{-1} A_{N} x_{N} \leq 1, \quad l_{N} \leq x_{N} \leq u_{N} .
\end{array}
$$

Clearly, this equivalent optimization problem is more compact and provides a better platform for the development of efficient algorithms.

In practice, conditions (2.2) do not need to be satisfied exactly. It suffices for $\hat{f}_{i}$ in (2.7) to be replaced by an interval $\left[l \hat{f}_{i}, u \hat{f}_{i}\right]$ for each $i \in\{1,2, \ldots, m\}$. By doing so, $\bar{C}$ turns out to be a so-called interval matrix given by

$$
\bar{C}=\left(\left[\underline{\bar{c}_{i j}}, \overline{\bar{c}_{i j}}\right]\right)
$$

where $\bar{c}_{i j}$ and $\overline{\bar{c}_{i j}}$ denote the lower and the upper bounds of the interval number $\bar{c}_{i j}$, respectively. Thus, the mathematical model for the optimal mixing design problem is expressed as the following linear program with interval coefficients:

$$
\begin{array}{ll}
\min & f(x) \\
\text { subject to } & \sum_{k=1}^{r}\left(\sum_{j=1}^{s}\left(\underline{c_{i j}}, \overline{\bar{c}_{i j}}\right) p_{k j}\right) x_{k}=0, \quad i=1,2, \ldots, m, \\
& \sum_{k=1}^{r} x_{k}=1, \quad l \leq x \leq u .
\end{array}
$$

For some $k \in\{1,2, \ldots, r\}$, the amounts of the ingredients $p_{k j}, j=1,2, \ldots, s$, of the $k$ th raw material could be probabilistic in nature. This is the case, for example, during the aluminium production process with sintering, because some raw materials are recycled. Thus, Problem (3.1) should be modified to give the following random linear program:

$$
\begin{array}{ll}
\min & f(x) \\
\text { subject to } & \bar{C} P^{(d)} x^{(d)}+\bar{C} P^{(p)} x^{(p)}=0, \quad \sum_{k=1}^{r} x_{k}=1, \quad l \leq x \leq u,
\end{array}
$$

where $P^{(d)}$ and $P^{(p)}$, which are the two submatrices of $P^{T}$, are partitioned in accordance with the deterministic and probabilistic columns of $P . x^{(d)}$ and $x^{(p)}$ are defined similarly. 
Combining (3.4) with (3.5), we obtain the following model for the mixture design problem:

$$
\begin{array}{ll}
\min & f(x) \\
\text { subject to } & \sum_{\substack{l \in I_{d}\\
}}\left(\sum_{j=1}^{s}\left(\underline{\bar{c}_{i j}}, \overline{\bar{c}_{i j}}\right) p_{l j}\right) x_{l}^{(d)}+\sum_{q \in I_{p}}\left(\sum_{j=1}^{s}\left(\underline{\bar{c}_{i j}}, \overline{\bar{c}_{i j}}\right) p_{q j}\right) x_{q}^{(p)}=0, \\
& \sum_{k=1}^{r} x_{k}=1, \quad l \leq x \leq u,
\end{array}
$$

where $I_{d}$ and $I_{p}$ are, respectively, the index sets of the deterministic and probabilistic columns of $P^{T}$, obtained from proper partitions of the set $\{1,2, \ldots, r\}$.

\section{Solution methods}

This section is devoted to the development of an efficient numerical solution method for solving the optimization model obtained above.

For a linear program (3.1), the software LINDO or the lp command in MATLAB can be used. However, for a linear program with interval or random coefficients, it cannot be solved directly as such. New solution methods need to be developed.

For a linear program with interval coefficients, the convexity of the feasible region is, in general, not valid. For example, consider the set

$$
\left\{\left[x_{1}, x_{2}\right]^{T}: x_{1}+x_{2}=0\right\} \subseteq \mathbb{R}^{2}
$$

which is clearly convex. However, the set

$$
\left\{\left[x_{1}, x_{2}\right]^{T}:[0.5,1] x_{1}+[2,1] x_{2}=0\right\}
$$

is nonconvex (see Figure 1) in $\mathbb{R}^{2}$. Fortunately, with the structural properties of model (3.4), we can prove that the feasible region of the problem (3.4) is convex.

Firstly, given a set $D$ with interval parameters

$$
D=\left\{\left[x_{1}, x_{2}\right]^{T}:[\underline{a}, \bar{a}] x_{1}+[\underline{b}, \bar{b}] x_{2}=0\right\},
$$

we prove the following two lemmas.

LEMMA 4.1. For given $[\underline{a}, \bar{a}]$ and $[\underline{b}, \bar{b}]$, let $\alpha=[\underline{a}, \bar{b}]^{T}, \beta=[\bar{a}, \underline{b}]^{T}$ and

$$
C=\left\{x \in \mathbb{R}^{2}: x=k_{1} \alpha+k_{2} \beta, k_{i} \in \mathbb{R}, k_{i} \geq 0, i=1,2\right\} .
$$

Then

$$
D=N_{C}\left(x^{(0)}\right)=\left\{\begin{array}{l|l}
x \in \mathbb{R}^{2} & \begin{array}{l}
\underline{a} x_{1}+\bar{b} x_{2} \leq 0, \\
\bar{a} x_{1}+\underline{b} x_{2} \geq 0, \\
x_{2} \geq 0
\end{array}
\end{array}\right\} \cup\left\{x \in \mathbb{R}^{2} \mid \begin{array}{l}
\frac{a}{\bar{a}} x_{1}+\bar{b} x_{2} \geq 0 \\
x_{2} \leq 0
\end{array}\right\}
$$




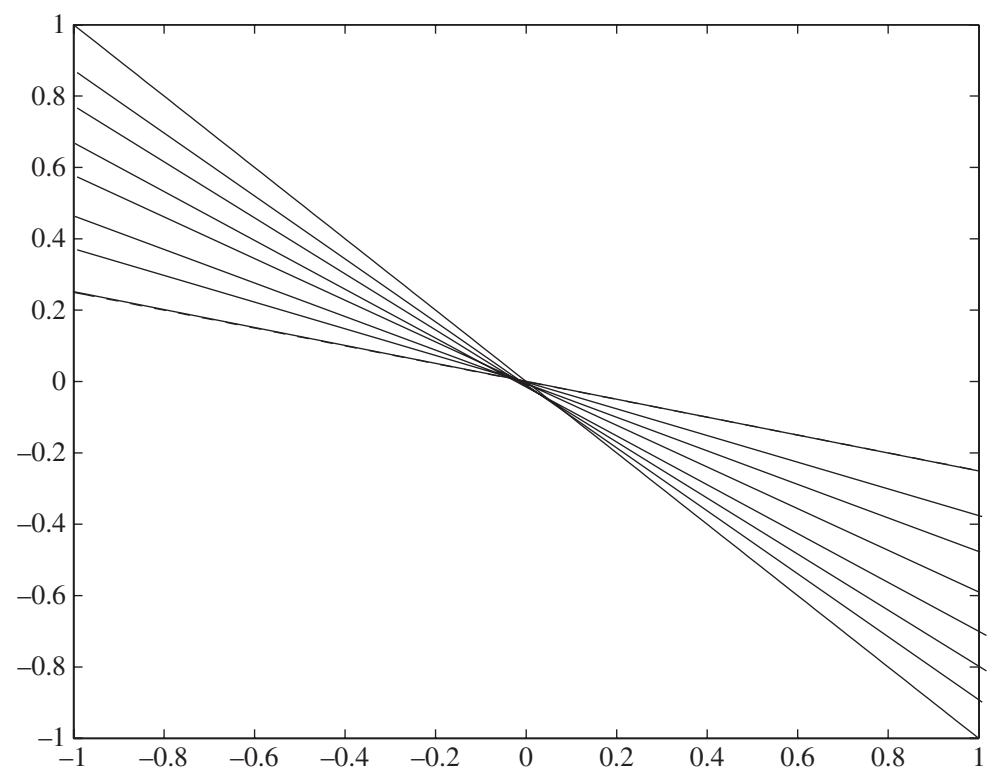

FIGURE 1. Nonconvex region determined by constraint (4.1) with interval coefficients.

where $x^{(0)}=[0,0]^{T}$ and $N_{C}\left(x^{(0)}\right)$ represents the normal cone to $C$ at $x^{(0)}$. Furthermore, except for the case of $\alpha=\beta$, the set $D$ is nonconvex.

Proof. Clearly, $x=\left[x_{1}, x_{2}\right]^{T} \in D$ if and only if there exist $a \in[\underline{a}, \bar{a}], b \in[\underline{b}, \bar{b}]$ such that

$$
a x_{1}+b x_{2}=0, \quad \text { that is, }[a, b]\left[\begin{array}{l}
x_{1} \\
x_{2}
\end{array}\right]=0 \text {. }
$$

Suppose $x_{2} \geq 0$. Then, by virtue of $\underline{a} \leq a$ and $\bar{b} \geq b$,

$$
[\underline{a}, \bar{b}]\left[\begin{array}{l}
x_{1} \\
x_{2}
\end{array}\right] \leq 0, \quad[\bar{a}, \underline{b}]\left[\begin{array}{l}
x_{1} \\
x_{2}
\end{array}\right] \geq 0 .
$$

Otherwise,

$$
[\underline{a}, \bar{b}]\left[\begin{array}{l}
x_{1} \\
x_{2}
\end{array}\right] \geq 0, \quad[\bar{a}, \underline{b}]\left[\begin{array}{l}
x_{1} \\
x_{2}
\end{array}\right] \leq 0 .
$$

Thus, the second equality in (4.2) holds.

By the definition of a normal cone, we obtain the first equality in (4.2). (See Figure 2.)

The last conclusion of Lemma 4.1 is obvious.

The conclusions of Lemma 4.1 can be extended to the $n$-dimensional case. Let

$$
D=\left\{x \in \mathbb{R}^{n}:\left[\underline{a_{1}}, \overline{a_{1}}\right] x_{1}+\left[\underline{a_{2}}, \overline{a_{2}}\right] x_{2}+\cdots+\left[\underline{a_{n}}, \overline{a_{n}}\right] x_{n}=0\right\} .
$$

Then we have the following lemma. 


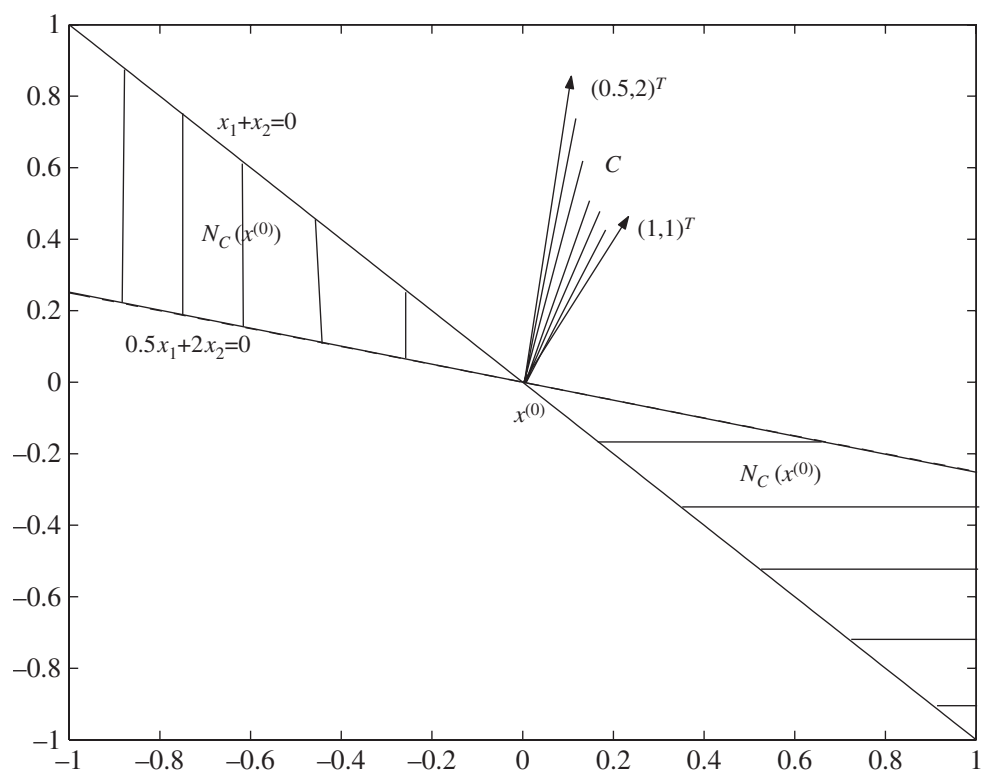

Figure 2. $D=N_{C}\left(x^{(0)}\right)$ defined by (4.2).

Lemma 4.2. For given $\left[\underline{a_{i}}, \overline{a_{i}}\right], i=1,2 \ldots, n$, let

$$
\begin{gathered}
\alpha=\left[\underline{a_{1}}, \underline{a_{2}}, \ldots, \underline{a_{n-1}}, \overline{a_{n}}\right]^{T}, \quad \beta=\left[\overline{a_{1}}, \overline{a_{2}}, \ldots, \overline{a_{n-1}}, \underline{a_{n}}\right]^{T}, \\
C=\left\{x \in \mathbb{R}^{n} \mid x=k_{1} \alpha+k_{2} \beta, k_{i} \in R, k_{i} \geq 0, i=1,2\right\} .
\end{gathered}
$$

Then

$$
\left.\begin{array}{rl}
D & =N_{C}\left(x^{(0)}\right) \\
& =\left\{\begin{array}{l|l}
\sum_{i=1}^{n-1} \underline{a}_{i} x_{i}+\overline{a_{n}} x_{n} \leq 0 \\
\sum_{i=1}^{n-1} \overline{a_{i}} x_{i}+\underline{a}_{n} x_{n} \geq 0, \\
x_{n} \geq 0
\end{array}\right.
\end{array}\right\} \cup\left\{\begin{array}{l|l}
x \in \mathbb{R}^{n} & \begin{array}{l}
\sum_{i=1}^{n-1} \underline{a}_{i} x_{i}+\overline{a_{n}} x_{n} \geq 0 \\
\sum_{i=1}^{n-1} \overline{a_{i}} x_{i}+\underline{a_{n}} x_{n} \leq 0, \\
x_{n} \leq 0
\end{array}
\end{array}\right\}
$$

where $x^{(0)}$ is the zero vector in $\mathbb{R}^{n}$. Furthermore, except for the case of $\alpha=\beta$, the set $D$ is nonconvex.

The proof of this lemma is similar to that given for Lemma 4.1, and hence is omitted.

In the following, we give an alternative approach to prove the second equality of the desired conclusion in Lemma 4.2. 
PROOF. $x \in D \subseteq \mathbb{R}^{n}$ if and only if there exists $a=\left[a_{1}, a_{2}, \ldots, a_{n}\right]^{T} \in \mathbb{R}^{n}$, where $a_{i} \in\left[\underline{a_{i}}, \overline{a_{i}}\right]$ for $i=1,2, \ldots, n$, such that

$$
a_{1} x_{1}+a_{2} x_{2}+\cdots+a_{n} x_{n}=0 .
$$

The value of the cosine angle of the normal vector $a$ of the hyperplane (4.3) with the axes $x_{n}$ is

$$
\cos \gamma=\frac{a_{n}}{\sqrt{a_{1}^{2}+a_{2}^{2}+\cdots+a_{n}^{2}}}
$$

Hence,

$$
\cos ^{2} \gamma=\frac{a_{n}^{2}}{a_{1}^{2}+a_{2}^{2}+\cdots+a_{n}^{2}}=\frac{1}{\left(a_{1}^{2}+\cdots+a_{n-1}^{2}\right) / a_{n}^{2}+1} .
$$

From (4.4), it follows readily that the maximal angle between $a$ and the positive direction of the axes $x_{n}$ is

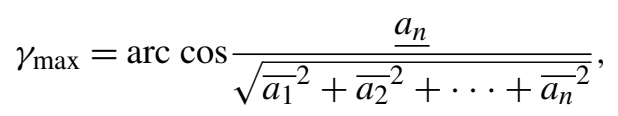

while the minimal angle is

$$
\gamma_{\min }=\arccos \frac{\overline{a_{n}}}{\sqrt{{\underline{a_{1}}}^{2}+\underline{a}_{2}^{2}+\cdots+\underline{a n}^{2}}} .
$$

By the continuity property, we know that the points on the hyperplane in $D$ are located in the set (see Figure 3)

$$
\begin{aligned}
& D=N_{C}\left(x^{(0)}\right)
\end{aligned}
$$

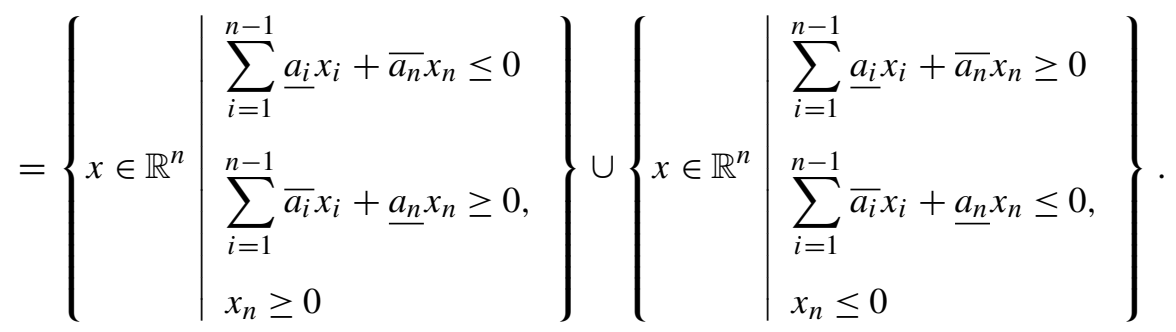

REMARK 4.3. With Lemma 4.2, and noting the nonnegative constraints $x_{n} \geq 0$ in (3.4), it can be seen that the feasible region of model (3.4) is a convex set defined by finite inequalities.

Actually, we have the following result.

THEOREM 4.4. Linear program (3.4) with interval equality constraints and nonnegative bound constraints is equivalent to an ordinary linear program only with finite inequality constraints and an equality. 


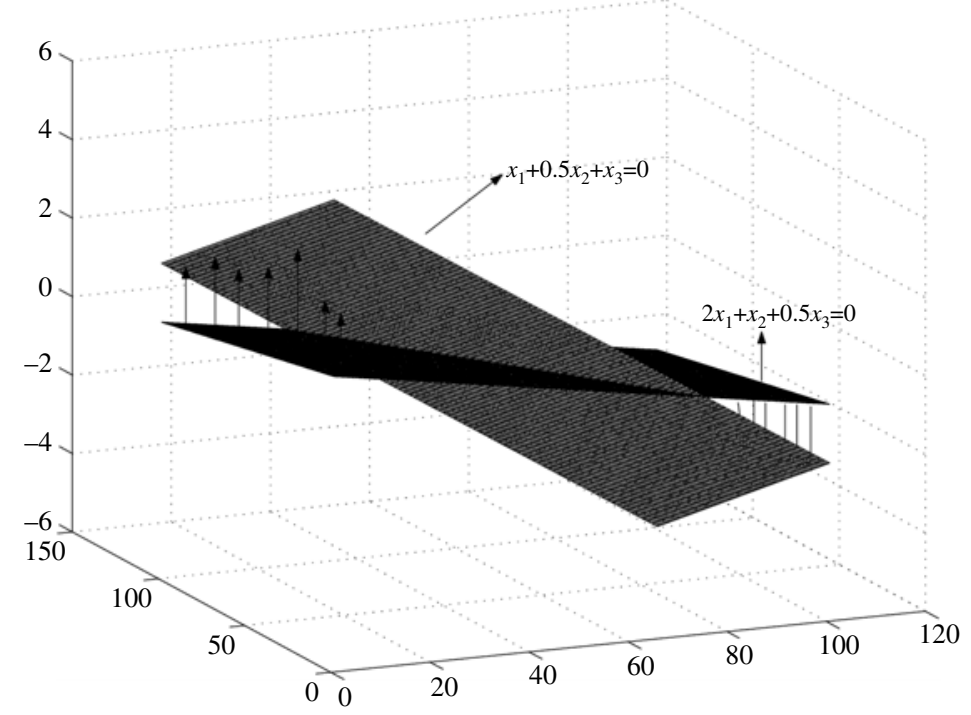

FIGURE 3. Nonconvex region in $\mathbb{R}^{3}$ determined by constraint (4.5) with interval coefficients.

Based on Theorem 4.4, linear programs with interval coefficients can be solved by the following algorithm.

Algorithm 1. Step 1. Write out the equivalent expression of each of the interval equality constraints in (3.4).

Step 2. Find the solution of the standard linear program obtained from Step 1.

We now move on to consider linear programs with probabilistic constraints given by

$$
\operatorname{Pr}\left\{\xi: h_{i}(x, \xi) \geq 0\right\} \geq p, \quad i=1,2, \ldots, m,
$$

where $\xi$ denotes the vector of random coefficients, and $p \in(0,1)$ is referred to as a probability level. These constraints are known in the literature as chance constraints; see, for example, [13]. They indicate that, for each $i=1,2, \ldots, m$, the probability of those $\xi$ such that the constraint

$$
h_{i}(x, \xi) \geq 0
$$

is satisfied is greater than or equal to $p$.

Suppose that the probability distribution function of the random vector $\xi$ is known. Then constraints (4.6) become

$$
\alpha_{i}(x) \geq p, \quad i=1,2, \ldots, m,
$$

where

$$
\alpha_{i}(x)=\operatorname{Pr}\left\{\xi: h_{i}(x, \xi) \geq 0\right\}, \quad i=1,2, \ldots, m .
$$

However, it is in general difficult to obtain explicit expressions for $\alpha_{i}(x), i=$ $1,2, \ldots, m$. Thus, the values and the derivatives of these functions are difficult to calculate. 
Assume, for example, that $h_{i}(x, \xi)$ is affine with respect to $x$, that is,

$$
h_{i}(x, \xi)=a_{i 1} x_{1}+a_{i 2} x_{2}+\cdots+a_{i n} x_{n}-b_{i} .
$$

However, $\alpha_{i}(x), i=1,2, \ldots, m$, are not necessary affine, and they are often nonconvex. Their deterministic equivalent formulations (DEFs), even if they can be obtained, are without some nice properties. See [13, 14, 18, 19] for details on the relevant issues. Here, we shall propose a new approach, called the satisfaction degree method, to a class of linear programs with random variables.

For clarity of presentation, we consider a linear program with only one constraint, whose coefficients are random variables:

$$
\begin{array}{ll}
\min & f(x) \\
\text { subject to } & a_{11} x_{1}+a_{12} x_{2}+\cdots+a_{1 n} x_{n} \leq b_{1},
\end{array}
$$

where $a_{1 j}$ and $b_{1}$ are random coefficients. The extension to multiple constraints is straightforward. The existing approaches to obtaining the deterministic equivalent formulation of (4.7) often require that the distribution functions of these coefficients are given and are statistically mutually independent. However, such requirements may not be satisfied in practice. The satisfaction degree approach to be introduced below is in response to the absence of these assumptions.

DEFINITION 4.5. For any random variables $x, y$, we call $\lambda(x \leq y)$, defined by

$$
\lambda(x \leq y)=\frac{E(y-x)}{\sqrt{D(y-x)}},
$$

the satisfaction degree of the random inequality $x \leq y$.

REMARK 4.6.

(1) In Definition 4.5, it is required that $D(y-x) \neq 0$. If $D(y-x)=0$, then $P(y-$ $x=E(y-x))=1$, which implies that $x$ is almost the same as $y$ regardless of the constant scalar.

(2) The possible values of $\lambda$ are as follows:

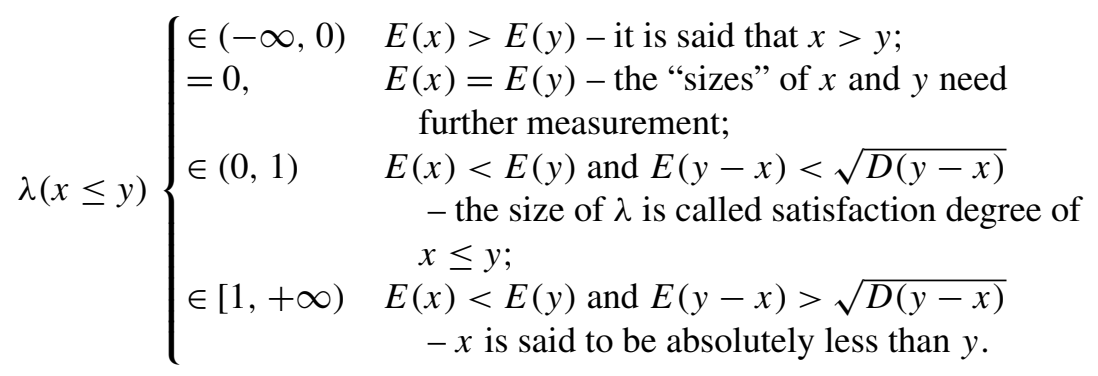

(3) If $\lambda(x \leq y)=0$ when $D(x)>D(y)$, it indicates that the deterministic degree of $y$ is greater than that of $x$. Thus, it is clear that $y$ is superior to $x$ for a decisionmaker.

(4) $\lambda(x \leq y) \leq 0$ is equivalent to $\lambda(y \leq x) \geq 0$. 
(5) If " $x \leq y$ " is regarded as equivalent to " $\lambda(x \leq y) \geq 0$ ", then such an ordering relation between two random numbers is of reflexivity and transitivity.

On the basis of Definition 4.5, a new deterministic equivalent formulation of random constraint is obtained.

THEOREM 4.7. For a given $\lambda_{0}$, the deterministic equivalent formulation of the random constraint in (4.7), that is,

$$
a_{11} x_{1}+a_{12} x_{2}+\cdots+a_{1 n} x_{n} \leq b_{1},
$$

obtained by the satisfaction degree approach is

$$
\mu z+\lambda_{0} \sqrt{z^{T} V z} \leq 0
$$

where $V$ denotes the covariance, $z^{T}=\left[x_{1}, \ldots, x_{n},-1\right]$, and $\mu=\left[\mu_{1}, \ldots, \mu_{n+1}\right]^{T}$ is the expectation of the random vector $\left[a_{11}, a_{12}, \ldots, a_{1 n}, b_{1}\right]^{T}$.

With Theorems 4.4 and 4.7, an efficient method is proposed in the next section for solving the mixture problems with uncertain parameters.

\section{Applications}

Consider a mixing design problem arising from the production process of aluminium with sintering. Due to the delay that occurs in the composition detection and the uncertainty that arises during the mixing process of the raw materials, the quality of the raw mineral slurry that comes out of the pipy grinder mixer may not satisfy the quality requirements for the consequent alumina sintering. For some relevant literature, see [5, 23, 24].

Consider a typical mixture problem, where there are six kinds of raw minerals, namely aluminiferous ore, lime, alkali, coal, alkali lye and silica slags. The mixture of these raw minerals is carried out in a pipy grinder mixer, where the raw mix slurry is formed. The goal is to determine the proportions $x_{r}$ of the raw minerals for $r=1,2,3,4,5,6$, such that certain quality indices are satisfied. In practice, the quality indices usually include the ratio of $\mathrm{Na}_{2} \mathrm{O}$ in both of $\mathrm{Al}_{2} \mathrm{O}_{3}$ and $\mathrm{Fe}_{2} \mathrm{O}_{3}$, the ratio of $\mathrm{CaO}$ in $\mathrm{SiO}_{2}$, the ratio of $\mathrm{Fe}_{2} \mathrm{O}_{3}$ in $\mathrm{Al}_{2} \mathrm{O}_{3}$, the ratio of $\mathrm{Al}_{2} \mathrm{O}_{3}$ in $\mathrm{SiO}_{2}$ and the ratio of water in the raw mix slurry. Here, the number of quality indices $m=3$. Let $\hat{f}_{i}$, $i=1,2,3$, denote the desired values of these three ratios.

Furthermore, we take

$$
\begin{gathered}
a_{11}=\frac{1}{62}, \quad a_{12}=a_{13}=a_{14}=a_{15}=0, \\
b_{12}=\frac{1}{102}, \quad b_{13}=\frac{1}{160}, \quad b_{11}=b_{14}=b_{15}=0 ; \\
a_{24}=\frac{1}{56}, \quad a_{21}=a_{22}=a_{23}=a_{25}=0 \\
b_{25}=\frac{1}{60}, \quad b_{21}=b_{22}=b_{23}=b_{24}=0 \\
a_{32}=\frac{1}{102}, \quad a_{31}=a_{33}=a_{34}=a_{35}=0 \\
b_{35}=\frac{1}{60}, \quad b_{31}=b_{32}=b_{33}=b_{34}=0 .
\end{gathered}
$$


Let $P \in \mathbb{R}^{r \times s}$ be the ingredient matrix, where $p_{k j}$ denotes the amount of the $j$ th ingredient in the $k$ th raw material. Then $r=6$ and $s=5$, and we obtain the matrix $C$ from (2.5) as

$$
\begin{aligned}
C & =\bar{C} P^{T}=\left[\begin{array}{cccccc}
a_{11} & -\hat{f}_{1} b_{12} & 0 & 0 & 0 & 0 \\
0 & 0 & 0 & a_{24} & -\hat{f}_{2} b_{25} & 0 \\
0 & a_{32} & 0 & 0 & -\hat{f}_{3} b_{35} & 0
\end{array}\right] P^{T} \\
& =\left[\begin{array}{cccc}
a_{11} p_{11}-\hat{f}_{1} b_{12} p_{12} & a_{11} p_{21}-\hat{f}_{1} b_{12} p_{22} & \cdots & a_{11} p_{61}-\hat{f}_{1} b_{12} p_{62} \\
a_{24} p_{14}-\hat{f}_{2} b_{25} p_{15} & a_{24} p_{24}-\hat{f}_{2} b_{25} p_{25} & \cdots & a_{24} p_{64}-\hat{f}_{2} b_{25} p_{65} \\
a_{32} p_{12}-\hat{f}_{3} b_{35} p_{15} & a_{32} p_{22}-\hat{f}_{3} b_{35} p_{25} & \cdots & a_{32} p_{62}-\hat{f}_{3} b_{35} p_{65}
\end{array}\right] .
\end{aligned}
$$

In practice, $\hat{f}_{i}, i=1,2,3$, should be interval parameters, while $p_{6 j}, j=$ $1,2, \ldots, 5$, should be random parameters. So, the constraints $C P^{T} x=0$ in model (3.1) should be replaced by

$$
\left\{\begin{array}{l}
\sum_{i=1}^{6}\left(a_{11} p_{i 1}-\left[\underline{\hat{f}_{1}}, \overline{\hat{f}_{1}}\right]\right) b_{12} p_{i 2} x_{i}=0 \\
\sum_{i=1}^{6}\left(a_{24} p_{i 4}-\left[\underline{\hat{f}_{2}}, \overline{\hat{f}_{2}}\right]\right) b_{25} p_{i 5} x_{i}=0 \\
\sum_{i=1}^{6}\left(a_{32} p_{i 2}-\left[\underline{\hat{f}_{3}}, \overline{\hat{f}_{3}}\right]\right) b_{35} p_{i 5} x_{i}=0 .
\end{array}\right.
$$

From model (3.6) and Lemma 4.2, the above mixture design problem with uncertain parameters should read

$$
\begin{array}{ll}
\min & f(x) \\
\text { such that } & \sum_{i=1}^{5}\left(a_{11} p_{i 1}-\underline{\hat{f}_{1}} b_{12} p_{i 2}\right) x_{i}+\left(a_{11} p_{61}-\overline{\hat{f}_{1}} b_{12} p_{62}\right) x_{6} \geq 0, \\
& \sum_{i=1}^{5}\left(a_{11} p_{i 1}-\overline{\hat{f}_{1}} b_{12} p_{i 2}\right) x_{i}+\left(a_{11} p_{61}-\underline{\hat{f}_{1}} b_{12} p_{62}\right) x_{6} \leq 0, \\
& \sum_{i=1}^{5}\left(a_{24} p_{i 4}-\underline{\hat{f}_{2}} b_{25} p_{i 5}\right) x_{i}+\left(a_{24} p_{64}-\overline{\hat{f}_{2}} b_{25} p_{65}\right) x_{6} \geq 0, \\
& \sum_{i=1}^{5}\left(a_{24} p_{i 4}-\overline{\hat{f}_{2}} b_{25} p_{i 5}\right) x_{i}+\left(a_{24} p_{64}-\underline{\hat{f}_{2}} b_{25} p_{65}\right) x_{6} \leq 0, \\
& \sum_{i=1}^{5}\left(a_{32} p_{i 2}-\underline{\hat{f}_{3}} b_{35} p_{i 5}\right) x_{i}+\left(a_{32} p_{62}-\overline{\hat{f}_{3}} b_{35} p_{65}\right) x_{6} \geq 0, \\
& \sum_{i=1}^{5}\left(a_{32} p_{i 2}-\overline{\hat{f}_{3}} b_{35} p_{i 5}\right) x_{i}+\left(a_{32} p_{62}-\underline{\hat{f}_{3}} b_{35} p_{65}\right) x_{6} \leq 0, \\
& \sum_{k=1}^{6} x_{k}=1, \quad l \leq x \leq u .
\end{array}
$$


Suppose that the distribution functions of $p_{6 j}, j=1,2, \ldots, 5$, are known. For example, let

$$
p_{6 j} \sim N\left(\mu_{j}, \sigma_{j}^{2}\right), \quad j=1,2,3,4,5 .
$$

Then, for a given confidence level $p$, the chance constraint

$$
\operatorname{Pr}\left\{\left(p_{61}, p_{62}\right) \mid \sum_{i=1}^{5}\left(a_{11} p_{i 1}-\underline{\hat{f}_{1}} b_{12} p_{i 2}\right) x_{i}+\left(a_{11} p_{61}-\overline{\hat{f}_{1}} b_{12} p_{62}\right) x_{6} \geq 0\right\} \geq p
$$

is equivalent to

$$
\begin{aligned}
& \left(a_{11} p_{11}-\underline{\hat{f}_{1}} b_{12} p_{12}\right) x_{1}+\cdots+\left(a_{11} p_{51}-\underline{\hat{f}_{1}} b_{12} p_{52}\right) x_{5} \\
& \quad+\left(E\left[a_{11} p_{61}-\overline{\hat{f}_{1}} b_{12} p_{62}\right]+\Phi^{-1}(p) \sqrt{V\left[a_{11} p_{61}-\overline{\hat{f}_{1}} b_{12} p_{62}\right]}\right) x_{6} \\
& \quad \leq 0
\end{aligned}
$$

where $E(\cdot)$ and $V[\cdot]$ represent, respectively, the expectation and the variance of the random variable, and $\Phi^{-1}$ is the inverse of standard normal distribution function.

Generally, it is true that

$$
E\left[a_{11} p_{61}-\overline{\hat{f}}_{1} b_{12} p_{62}\right]=a_{11} E\left[p_{61}\right]-\overline{\hat{f}_{1}} b_{21} E\left[p_{62}\right]=a_{11} \mu_{1}-\overline{\hat{f}}_{1} b_{21} \mu_{2},
$$

and

$$
\begin{aligned}
V\left[a_{11} p_{61}-\overline{\hat{f}_{1}} b_{12} p_{62}\right]= & a_{11}^{2} E\left[p_{61}^{2}\right]+\left(\overline{\hat{f}_{1}} b_{12}\right)^{2} E\left[p_{62}^{2}\right]-2 a_{11} \overline{\hat{f}_{1}} b_{12} E\left[p_{61} p_{62}\right] \\
& -\left(a_{11} \mu_{1}-\overline{\hat{f}_{1}} b_{21} \mu_{2}\right)^{2} .
\end{aligned}
$$

Furthermore, under the assumption that $p_{61}$ and $p_{62}$ are two mutually independent random variables,

$$
V\left[a_{11} p_{61}-\overline{\hat{f}}_{1} b_{12} p_{62}\right]=a_{11}^{2} \sigma_{1}^{2}+\left(\overline{\hat{f}_{1}} b_{21}\right)^{2} \sigma_{2}^{2} .
$$

Thus, (5.2) can be written as

$$
\begin{aligned}
& \sum_{i=1}^{5}\left(a_{11} p_{i 1}-\underline{\hat{f}_{1}} b_{12} p_{i 2}\right) x_{i} \\
& \quad+\left(a_{11} \mu_{1}-\overline{\hat{f}_{1}} b_{21} \mu_{2}+\Phi^{-1}(p) \sqrt{a_{11}^{2} \sigma_{1}^{2}+\left(\overline{\hat{f}_{1}} b_{21}\right)^{2} \sigma_{2}^{2}}\right) x_{6} \leq 0 .
\end{aligned}
$$

Similarly to how we deal with the first chance constraint, we can obtain the deterministic equivalent linear expressions of the other five chance constraints in (5.1). Therefore, the chance constrained program (5.1) is equivalent to an ordinary linear program. Solving such a linear programming problem, we obtain the solution of the original mixture problem with uncertain parameters. 
In the case where $P_{6 i}, i=1,2,3,4,5,6$, are not mutually independent random variables with unknown distributions except that their expectation and covariance can be evaluated from sampling, the satisfaction degree method proposed in this paper can find the deterministic equivalent formulation of the constructed random model.

Denote

$$
\begin{aligned}
& c_{61}=\overline{\hat{f}_{1}} b_{12} p_{62}-a_{11} p_{61}, \quad c_{62}=a_{11} p_{61}-\underline{\hat{f}_{1}} b_{12} p_{62}, \quad c_{63}=\overline{\hat{f}_{2}} b_{25} p_{65}-a_{24} p_{64}, \\
& c_{64}=a_{24} p_{64}-\underline{\hat{f}_{2}} b_{25} p_{65}, \quad c_{65}=\overline{\hat{f}_{3}} b_{35} p_{65}-a_{32} p_{62}, \quad c_{66}=a_{32} p_{62}-\underline{\hat{f}_{3}} b_{35} p_{65} .
\end{aligned}
$$

Note that

$$
\begin{aligned}
& E\left(c_{61}\right)=\overline{\hat{f}_{1}} b_{12} E\left(p_{62}\right)-a_{11} E\left(p_{61}\right), \\
& D\left(c_{61}\right)=\left(\overline{\hat{f}_{1}} b_{12}\right)^{2} D\left(p_{62}\right)+a_{11}^{2} D\left(p_{61}\right)-2 \overline{\hat{f}_{1}} b_{12} a_{11} \operatorname{cov}\left(p_{61}, p_{62}\right),
\end{aligned}
$$

where $E(\cdot)$ represents the expectation, $D(\cdot)$ denotes the variance, and $\operatorname{cov}(\cdot)$ denotes the covariance.

From the satisfaction degree method, the deterministic equivalent formulation of the first random inequality constraint in (5.1) is given by

$$
\sum_{i=1}^{5}\left(\underline{\hat{f}_{1}} b_{12} p_{i 2}-a_{11} p_{i 1}\right) x_{i}+\left(E\left(c_{1}\right)+\lambda \sqrt{D\left(c_{1}\right)}\right) x_{6} \leq 0,
$$

where $\lambda$ is the satisfaction degree.

Similarly, we obtain

$$
\begin{aligned}
& E\left(c_{62}\right)=a_{11} E\left(p_{61}\right)-\underline{\hat{f}_{1} b_{12} E\left(p_{62}\right),} \\
& D\left(c_{62}\right)=a_{11}^{2} D\left(p_{61}\right)+\left(\underline{\hat{f}_{1}} b_{12}\right)^{2} D\left(p_{62}\right)-2 \underline{\hat{f}_{1}} b_{12} a_{11} \operatorname{cov}\left(p_{61}, p_{62}\right) ; \\
& E\left(c_{63}\right)=\overline{\hat{f}_{2}} b_{25} E\left(p_{65}\right)-a_{24} E\left(p_{64}\right), \\
& D\left(c_{63}\right)=\left(\overline{\hat{f}_{2}} b_{25}\right)^{2} D\left(p_{65}\right)+a_{24}^{2} D\left(p_{64}\right)-2 \hat{\hat{f}}_{2} b_{25} a_{24} \operatorname{cov}\left(p_{64}, p_{65}\right) ; \\
& E\left(c_{64}\right)=a_{24} E\left(p_{64}\right)-\underline{\hat{f}_{2}} b_{25} E\left(p_{65}\right), \\
& D\left(c_{64}\right)=a_{24}^{2} D\left(p_{64}\right)+\left(\overline{\hat{f}_{2}} b_{25}\right)^{2} D\left(p_{65}\right)-2 \hat{\hat{f}}_{25} b_{24} \operatorname{cov}\left(p_{64}, p_{65}\right) ; \\
& E\left(c_{65}\right)=\overline{\hat{f}_{3}} b_{35} E\left(p_{65}\right)-a_{32} E\left(p_{62}\right), \\
& D\left(c_{65}\right)=\left(\overline{\hat{f}_{3}} b_{35}\right)^{2} D\left(p_{65}\right)+a_{32}^{2} D\left(p_{62}\right)-2 \underline{\hat{f}_{3}} b_{35} a_{32} \operatorname{cov}\left(p_{62}, p_{65}\right) ; \\
& E\left(c_{66}\right)=a_{32} E\left(p_{62}\right)-\underline{\hat{f}_{3}} b_{35} E\left(p_{65}\right), \\
& D\left(c_{66}\right)=a_{32}^{2} D\left(p_{62}\right)+\left(\underline{\hat{f}_{3}} b_{35}\right)^{2} D\left(p_{65}\right)-2 b_{35} a_{32} \operatorname{cov}\left(p_{62}, p_{65}\right) .
\end{aligned}
$$


Thus, the deterministic equivalent formulation of the original model with interval and random coefficients is

$$
\begin{array}{ll}
\min & f(x)=p^{T} x \\
\text { s.t. } & \sum_{i=1}^{5}\left(\underline{\hat{f}_{1}} b_{12} p_{i 2}-a_{11} p_{i 1}\right) x_{i}+\left(E\left(c_{16}\right)+\lambda \sqrt{D\left(c_{16}\right)}\right) x_{6} \leq 0, \\
& \sum_{i=1}^{5}\left(a_{11} p_{i 1}-\overline{\hat{f}_{1}} b_{12} p_{i 2}\right) x_{i}+\left(E\left(c_{26}\right)+\lambda \sqrt{D\left(c_{26}\right)}\right) x_{6} \leq 0, \\
& \sum_{i=1}^{5}\left(\underline{\hat{f}_{2}} b_{25} p_{i 5}-a_{24} p_{i 4}\right) x_{i}+\left(E\left(c_{36}\right)+\lambda \sqrt{D\left(c_{36}\right)}\right) x_{6} \leq 0, \\
& \sum_{i=1}^{5}\left(a_{24} p_{i 4}-\overline{\hat{f}_{2}} b_{25} p_{i 5}\right) x_{i}+\left(E\left(c_{46}\right)+\lambda \sqrt{D\left(c_{46}\right)}\right) x_{6} \leq 0, \\
& \sum_{i=1}^{5}\left(\underline{\hat{f}_{3}} b_{35} p_{i 5}-a_{32} p_{i 2}\right) x_{i}+\left(E\left(c_{56}\right)+\lambda \sqrt{D\left(c_{56}\right)}\right) x_{6} \leq 0, \\
& \sum_{i=1}^{5}\left(a_{32} p_{i 2}-\overline{\hat{f}_{3}} b_{35} p_{i 5}\right) x_{i}+\left(E\left(c_{66}\right)+\lambda \sqrt{D\left(c_{66}\right)}\right) x_{6} \leq 0, \\
& x_{1}+x_{2}+x_{3}+x_{4}+x_{5}+x_{6}=1, \quad l_{i} \leq x_{i} \leq u_{i}, \quad i=1,2, \ldots, 6 .
\end{array}
$$

Clearly, model (5.3) is a standard linear program. Thus, it can be solved by standard techniques for linear programs.

\section{Numerical examples}

In this section, we adapt the methods developed in Section 4 to solve the optimization problems arising from the mixing design in the production process of aluminium with sintering.

Assume that the ingredients of the stable raw materials are given by

$$
\left[\begin{array}{ccccc}
0 & 0.5 & 0.35 & 0.15 & 0.2 \\
0.02 & 0.54 & 0.01 & 0.15 & 0 \\
0.30 & 0.25 & 0.10 & 0 & 0.03 \\
0 & 0.05 & 0 & 0 & 0.02 \\
0 & 0 & 0 & 0.03 & 0.08
\end{array}\right]
$$

which consists of the submatrix of the first five rows in $P$. The last row of $P$, denoted as $P_{6 \bullet}$, is a random vector with expected value

$$
E\left(p_{61}, p_{62}, p_{63}, p_{64}, p_{65}\right)=\left[\begin{array}{lllll}
0.1067 & 0.1950 & 0.0900 & 0.2117 & 0.1550
\end{array}\right],
$$

and variance

$$
D\left(p_{61}, p_{62}, p_{63}, p_{64}, p_{65}\right)=\left[\begin{array}{lllll}
0.0006 & 0.0007 & 0.0006 & 0.0026 & 0.0011
\end{array}\right]^{T} .
$$


The covariance matrix of $P_{6 \bullet}$ is

$$
V=\left[\begin{array}{rrrrr}
0.0006 & -0.0003 & 0.0005 & -0.0006 & 0.0001 \\
-0.0003 & 0.0007 & -0.0004 & -0.0002 & 0.0001 \\
0.0005 & -0.0004 & 0.0006 & -0.0006 & 0.0000 \\
-0.0006 & -0.0002 & -0.0006 & 0.0026 & -0.0001 \\
0.0001 & 0.0001 & 0.0000 & -0.0001 & 0.0011
\end{array}\right]
$$

Suppose that the price vector $p=\left[\begin{array}{llllll}1 & 0.3 & 1.5 & 0.95 & 0.8 & 1.2\end{array}\right]^{T}$. Set

$$
l=\left[\begin{array}{llllll}
0.01 & 0.01 & 0 & 0 & 0 & 0.04
\end{array}\right]^{T}, \quad u=\left[\begin{array}{llllll}
0.25 & 0.3 & 0.3 & 0.7 & 0.4 & 0.6
\end{array}\right]^{T} .
$$

Assume that the three interval coefficients are

$$
\hat{f}_{1}=[0.15,0.4], \quad \hat{f}_{1}=[0.32,0.85], \quad \hat{f}_{1}=[0.5,0.7] .
$$

Given the satisfaction degree $\lambda=0.5$, then by solving (5.3), the solution of the original model with interval and random parameters is obtained.

In the above setting, using the lp command in MATLAB, we obtain the optimal solution

$$
x^{*}=\left[\begin{array}{lllllll}
0.01 & 0.05185897 & 0.07414184 & 0.4239992 & 0.4 & 0.04
\end{array}\right]^{T} .
$$

The minimal cost $f^{*}=0.9075697$.

Taking $\lambda=0.99$, we obtain the optimal solution

$$
x^{*}=\left[\begin{array}{lllllll}
0.01 & 0.04987091 & 0.07593617 & 0.4241929 & 0.3600769 & 0.04
\end{array}\right]^{T},
$$

and the minimal cost $f^{*}=0.9098488$, which is greater than that for $\lambda=0.5$.

\section{Final remarks}

In this paper, we have formulated a mixture making problem as a new class of linear programs, where there are only equality constraints with interval or random coefficients in addition to box constraints. We have also presented some admissibility conditions for this class of problems.

In the models constructed, we have taken into account the uncertainty effects that often occur during the production process. Efficient methods fully utilizing the specific features of the models were presented. It was shown that these models are reducible, and can be reformulated as linear or nonlinear deterministic optimization programs.

We have applied the models and solution methods to a mixing proportion problem arising from aluminium production with sintering. Numerical tests showed that the models and the solution methods are promising. 


\section{Acknowledgements}

This work is supported by the National Natural Science Fund of China (grant no. 60804037) and the project for Excellent Talent of New Century, Ministry of Education, China (grant no. NCET-07-0864).

\section{References}

[1] J. E. Astier, "Main development in iron ore preparation", in: The Second International Conference on the Iron Ore Industry (Luleå University, Kiruna, Sweden, 2002).

[2] A. Ben-Tal, A. Goryashko, E. Guslitzer and A. Nemirovski, "Adjustable robust solutions of uncertain linear programs", Math. Program. 99(2, Ser. A) (2004) 351-376.

[3] A. Berkelaar, C. Dert, B. Oldenkamp and S. Zhang, "A primal-dual decomposition-based interior point approach to two-stage stochastic linear programming”, Oper. Res. 50 (2002) 904-915.

[4] P. K. Chang, "An approach to optimizing mix design for properties of high-performance concrete", Cement Concrete Res. 34(4) (2004) 623-629.

[5] H. W. Chen, "Improvement on burden calculation for raw mix slurry in production of alumina with sintering process", World Nonferrous Metals 12 (2001) 36-41.

[6] D. Dentcheva, B. Lai and A. Ruszczynski, "Dual methods for probabilistic optimization problems", Math. Methods Oper. Res. 60 (2004) 331-346.

[7] D. Dentcheva, A. Prékopa and A. Ruszczyński, "Concavity and efficient points of discrete distributions in probabilistic programming”, Math. Program. 89(1, Ser. A) (2000) 55-77.

[8] C. A. DePaolo and J. Rader, "A heuristic algorithm for a chance constrained stochastic program", European J. Oper. Res. 176 (2007) 27-45.

[9] Y. Ermoliev and R. J.-B. Wets, Numerical techniques for stochastic optimization (Springer, Berlin, 1988).

[10] B. Felekoğlu, S. Türkel and B. Baradan, "Effect of water/cement ratio on the fresh and hardened properties of self-compacting concrete", Building and Environment 42(4) (2007) 1795-1802.

[11] L. H. Hsieh and J. A. Whiteman, "Effect of raw material composition on the mineral phases in lime-fluxed iron ore sinter", ISIJ International 33(4) (1993) 462-473.

[12] T. Ji, T. Lin and X. Lin, "A concrete mix proportion design algorithm based on artificial neural networks", Cement Concrete Res. 36(7) (2006) 1399-1408.

[13] P. Kall, Stochastic linear programming (Springer, Berlin, 1976).

[14] C. M. Lagoa, X. Li and M. Sznaier, "Probabilistically constrained linear programs and riskadjusted controller design", SIAM J. Optim. 15 (2005) 938-951.

[15] C. H. Lim, Y. S. Yoon and J. H. Kim, "Genetic algorithm in mix proportioning of high-performance concrete", Cement Concrete Res. 34(3) (2004) 409-420.

[16] X. X. Lin, S. L. Janak and C. A. Floudas, "New robust optimization approach for scheduling under uncertainty I: Bounded uncertainty”, Comput. Chemical Eng. 28 (2004) 1069-1085.

[17] M. K. Luhandjula, "Fuzzy stochastic linear programming: Survey and future research directions", European J. Oper. Res. 174(3) (2006) 1353-1367.

[18] J. Mayer, Stochastic linear programming algorithms (Gordon and Breach, Amsterdam, 1998).

[19] A. Ruszczynski and A. Shapiro, "Stochastic programming", in: Handbook in operations research and management science (Elsevier, Amsterdam, 2003).

[20] N. Su, K. Hsu and H. Chai, "A simple mix-design method for selfcompacting concrete", Cement Concrete Res. 31 (2001) 1799-1807.

[21] M. T. Vieira, L. Catarino, M. Oliveira, J. Sousa, J. M. Torralba, L. E. G. Cambronero, F. L. González-Mesones and A. Victoria, "Optimization of the sintering process of raw material wastes", J. Materials Processing Technology 93 (1999) 97-101.

[22] D. Q. Wang, "Application of linear programming in production of mixing materials to sinter", China Metallurgy 15(8) (2005) 19-22. 
[23] C. H. Yang, X. G. Duan, Y. L. Wan and W. H. Gui, "Blending expert system for raw mix slurry in production of alumina with sintering process", J. Cent. South Univ. (Science and Technology) 36(4) (2005) 648-652.

[24] X. K. Yang, C. H. Yang, Y. L. Wang and W. H. Gui, "The application of improved genetic algorithm in raw mix slurry optimal arrangement of alumina process", Computing Technology and Automation 25(1) (2006) 44-46.

[25] M. F. M. Zain, Md. N. Islam and Ir. H. Basri, "An expert system for mix design of high performance concrete", Adv. Engineering Software 36(5) (2005) 325-337. 